\title{
Schweizerische Gesellschaft für Notfall- und Rettungsmedizin SGNOR
}

Gabriela Kaufmann-Hostettler

Geschäftsführerin SGNOR

Korrespondenz:

Zentralsekretariat SGNOR SSMUS c/o gkaufmann Verbandsmanagement Wattenwylweg 21 CH-3006 Bern

sekretariat@sgnor.ch

www.sgnor.ch

www.ssmus.ch

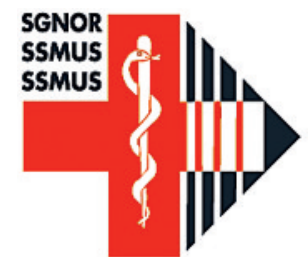

Die SGNOR wurde im Jahr 1990 als Vereinigung Schweizer Notärzte VSN im Anschluss an einen Notarztkurs in Nottwil gegründet und zählte anfänglich um die 100 Mitglieder. Eine erste - über die Grenzen der Notfallmedizin hinausgehende - Anerkennung erlebte sie durch den durch die FMH in Kraft gesetzten Fähigkeitsausweis Notarzt SGNOR am 1. Januar 2000. Dieser Ausweis basiert auf der Weiterbildungsordnung der Verbindung der Schweizer Ärztinnen und Ärzte FMH. Die SGNOR ist verantwortlich für die Einhaltung der Vorgaben, sie hat die Qualität der Weiterbildung zu überprüfen. Inzwischen gehören der SGNOR rund 700 Mitglieder an.

\section{Gewachsene Aufgaben, starke Stimme}

Mit dem Einbezug der klinischen Notfallmedizin sowie der Katstrophenmedizin (beschlossen durch die Mitgliederversammlung SGNOR im Jahr 2005) sind die Aufgaben der SGNOR gewaltig gewachsen. Der Vorstand wurde aus diesem Grund von 9 auf 13 Mitglieder erhöht. Im weiteren gibt es in der SGNOR eine Bildungs- und eine Wissenschaftskommission. Das Forum klinische Notfallmedizin arbeitet zudem eng mit der SGNOR zusammen.

Die SGNOR hat seit 2006 in der Ärztekammer Mitsprache- und Antragsrecht. Sie ist Mitglied des Interverbands für Rettungswesen IVR, der Swiss Medical Rescue Commission SMEDREC, des Swiss Resuscitation Councils SRC, des Forums Berufsbildung Rettungswesen - und auf internationaler Ebene - der European Society for Emergency Medicine EuSEM.

\section{Engagiert in der Weiterbildung}

Jährlich stellt die SGNOR an die 200 Diplome aus, seien dies neue Fähigkeitsausweise Notarzt SGNOR oder Rezertifizierungen desselben, aber auch Bestätigungen des Programms Klinische Notfallmedizin SGNOR. Ein weiteres, wichtiges
Tätigkeitsfeld der SGNOR ist das Kurswesen. Sie bietet die folgenden Kurse an, an denen jährlich rund 1500 Personen teilnehmen: Dienstarztkurs SGNOR, Notarztkurs SGNOR, ACLS-AHA (Advanced Cardiac Life Support der American Heart Association) sowie PALS-AHA (Pediatric Advanced Life Support der American Heart Association).

\section{Aktuell}

Am 11. Dezember 2008 haben die Delegierten der ausserordentlichen Ärztekammer der Schaffung des Fähigkeitsausweises Klinische Notfallmedizin SGNOR mit überwältigendem Mehr zugestimmt. Für die SGNOR ist dies ein wichtiger Meilenstein, hat sie doch vier Jahre für diesen Fähigkeitsausweis gekämpft. Der Vorstand der SGNOR dankt an dieser Stelle all jenen, die zu diesem Erfolg beigetragen haben, und freut sich auf die Umsetzung des Programms.

\section{Vorstand}

Präsident:

Dr. med. Ulrich Bürgi, Aarau

Vizepräsident:

Dr. med. Luciano Anselmi, Bellinzona

Aktuar:

Dr. med. Gregor Niedermaier, Davos

Past-President:

Dr. med. Lion Bernoulli, Zürich

Kommunikation:

Dr. med. Roland Albrecht, Zürich

Finanzen:

Dr. med. Zeno Supersaxo, Thun

Wissenschaft:

Prof. Dr. med. Bernard Vermeulen, Freiburg

Kurse:

Dr. med. Dieter von Ow, St. Gallen

Mitglieder:

Prof. Dr. med. Roland Bingisser, Basel;

Dr. med. Beat Lehmann, Freiburg;

Dr. med. Peter Rupp, Bern;

Prof. Dr. med. François Sarasin, Genf;

Dr. med. Robert Sieber, Lugano 